surface of the flake remains unimpaired throughout the oxidation; there is no pitting of this surface. Apparently carbon atoms are removed layer by layer. (b) The holes which eventually appear in the flake have a well-defined hexagonal form, which they retain as they grow, although when they reach the size shown, the corners begin to round off a little; when smaller, the hexagon appeared very regular. (Unfortunately, the reproductions appear more distorted than the original photographs, themselves imperfect.) The shadows round the flake in Fig. 1, $B$, are caused by semi-opaque patches in the silica pan carrying the flake. It will be noticed that corresponding sides of the two clearly formed hexagons are parallel.

Possibly the formation of these holes is caused in the first place by the presence of minute inclusions of impurity, such as iron or an alkali metal salt, which would catalyse the oxidation of the carbon with which it is in contact. But the subsequent growth of the hexagons provides a manifestation of the influence of lattice symmetry upon the course of a chemical reaction. It seems to us that the points $(a)$ and $(b)$ are of interest in connexion with the mechanism of the oxidation of graphite.

E. N. Greer. B. TOPLEY.

The Sir William Ramsay Laboratories of

Inorganic and Physical Chemistry, University College, London, W.C.1, May 12.

\section{Oxidation of Strychnine}

I HAVE been studying the action of oxidising agents on strychnine for some time. Leuchs ${ }^{1}$ stated that he had obtained an acid with the formula $\mathrm{C}_{21} \mathrm{H}_{22} \mathrm{O}_{4} \mathrm{~N}_{2}$ from strychnidine by means of oxidation with chromic acid. The acid is stated to crystallise from water in brown needles that do not melt up to $320^{\circ}$, and are nearly insoluble in alcohol and acetone, but readily soluble in ammonia, $N$-hydrochloric acid, and acetic acid. The acid in question gives crystalline precipitates in needles and prisms with nitric and hydrobromic acids, and amorphous precipitates with hydriodic and perchloric acids, which then become crystalline scales.

It appears that Leuchs ${ }^{2}$ obtained this same compound by oxidising tetra-hydro-strychnine with large quantities of chromic acid in a carrier acidified with sulphuric acid, and Wieland admits that the high melting point of this compound shows ${ }^{3}$ that the acid $\mathrm{C}_{21} \mathrm{H}_{22} \mathrm{O}_{4} \mathrm{~N}_{2}$ is a diphenyl derivate, to which he attributes, with more probability, the formula of $\mathrm{C}_{42} \mathrm{H}_{42} \mathrm{~N}_{4} \mathrm{O}_{8}{ }^{4}$

I would like to point out that by oxidising strychnine with chromic acid in a carrier acidified with acetic acid, I have obtained a crystalline compound in very shiny prisms, which melt at $309^{\circ}-310^{\circ}$, and are soluble in alcohol and chymol when hot, but practically insoluble in water and all organic solvents, especially chloroform.

The reaction of this compound is neutral with litmus paper, and it gives strongly the colour reactions of strychnine. The product in question is obtained with several processes, and analysis gave the following results :

Microscopic analysis gave the following percentages : Carbon- $68 \cdot 77,68 \cdot 69,69 \cdot 07,68 \cdot 58$. Hydrogen-- $5 \cdot 68,5 \cdot 65,5 \cdot 61,5 \cdot 53$. Nitrogen- $7 \cdot 31,7 \cdot 38,7 \cdot 85,7 \cdot 75$.

Macroscopic analysis gave the following percentages : Carbon- 68.99, 68.86.

Hydrogen $-5 \cdot 75, \quad 5 \cdot 88$.

Nitrogen- 7:40.

These results agree both with a formula of No. 3268, VoL. 129]
$\mathrm{C}_{21} \mathrm{H}_{20} \mathrm{~N}_{2} \mathrm{O}_{4}$ and that of $\mathrm{C}_{42} \mathrm{H}_{42} \mathrm{~N}_{4} \mathrm{O}_{8}$, and they seem to $\mathrm{me}$ to be interesting, since $\mathrm{I}$ am of the opinion that this is the first case in which a product has been obtained from the oxidation of strychnine that has the same number of atoms of carbon as that of the alkaloid itself.

Fuller details regarding this question will be sent later to the Gazzetta Chimica Italiana.

Istituto Chem. Farm., Bernardi Alessandro.

R. Università,

Bologna, March 29.

1 Ber., 63, 2215 ; 1930.

\& Ber., 63, $3184 ; 1930$.

L Lieb. Ann., 491, 111; 1931.
Ber., 65, 201; 1932.

\section{The $\mathrm{NO}_{3}$ Frequency in Organic Nitrates}

The characteristic inactive frequency at $9.5 \mu$, corresponding to a wave-number shift of $1050 \mathrm{~cm} .^{-1}$, while it appears as a strong Raman line in the scattered spectrum of all inorganic nitrates examined both as crystals and in solutions, is not represented at all in the Raman effect of the organic nitrates. This is probably due to the influence of the $\mathrm{CH}$ group on the oscillations of the $\mathrm{NO}_{3}$ ion. However, in alcoholic solutions of these organic nitrates this line comes out significantly, the intensity of the line depending upon the solvent used and also upon the concentration of the solution.

In the scattered spectrum of methyl nitrate (pure liquid), in addition to the $\mathrm{CH}$ frequencies, the observed Raman lines correspond to $d v 583,662,866$, and 1290 , all of which are quite strong. These shifts, which are simple in methyl nitrate, are each split up into two or more components in the spectra of the higher alkyl nitrates, and also there is a considerable change in their intensities. In a twenty per cent solution of methyl nitrate in methyl alcohol none of these lines comes out, but instead we get a strong line at $\nu 23,665 \mathrm{~cm} .^{-1}$ which is excited by $\lambda 4047$ of the mercury are and corresponds to a shift of 1040 . An equimolecular solution of amyl nitrate in amyl alcohol shows this shift only as a very faint line.

In this connexion it may be mentioned that the dielectric constant of methyl alcohol is very high, coming next to water, with a value 35 , while for amyl alcohol it is only 16 . We have studied the behaviour of this shift with dilution and there is a general tendency for the line to increase in intensity as the dilution increases up to a certain limit. A more detailed acount of the investigation appears elsewhere.

A. S. Ganesan.

College of Science, Nagpur, India, April 28.

\section{A Displacement in the $N$ Lines of Tungsten and Tantalum}

WE have recently conducted some experiments on the characteristic radiations of various elements in the extreme soft $\mathrm{X}$-ray region. Spectra have been obtained with the aid of a vacuum spectrograph employing a plane Siegbahn grating in the grazing incidence setting. Using a water-cooled anti-cathode, on to which tungsten was sputtered from the hot filament of the X-ray tube, we have obtained lines at 55.58 and $58.42 \mathrm{~A}$. Thibaud and Soltan ${ }^{1}$ have previously obtained wave-lengths of 56.0 and $59 \cdot 1 \mathrm{~A}$., while Prins, ${ }^{2}$ who, in addition to using this procedure, has apparently employed a water-cooled tungsten anticathode, has recently published values of $55 \cdot 7$ and $58.4 \mathrm{~A}$., with which our results are in good accord. 